\title{
Uncovering Consumer Mindsets Regarding Raw Beverages
}

\author{
Ryan Zemel ${ }^{1}$, Attila Gere ${ }^{2}$, Petraq Papajorgji ${ }^{3}$, Glenn Zemel ${ }^{4}$, Howard Moskowitz ${ }^{5}$ \\ ${ }^{1}$ Mind Genomics Associates, Inc., Downers Grove, USA \\ ${ }^{2}$ Department of Postharvest and Sensory Evaluation, Sensory Laboratory, Faculty of Food Science, Szent István University, \\ Budapest, Hungary \\ ${ }^{3}$ Department of Computer Science, University of New York Tirana, Tirana, Albania \\ ${ }^{4}$ Mind Genomics Associates, Inc., White Plains, New York, USA \\ ${ }^{5}$ The Big Mind Institute for Education and Messaging, White Plains, New York, USA \\ Email: rzemel@icloud.com
}

How to cite this paper: Zemel, R., Gere, A., Papajorgji, P., Zemel, G. and Moskowitz, H. (2018) Uncovering Consumer Mindsets Regarding Raw Beverages. Food and Nutrition Sciences, 9, 259-267. https://doi.org/10.4236/fns.2018.93020

Received: January 18, 2018

Accepted: March 27, 2018

Published: March 30, 2018

Copyright (c) 2018 by authors and Scientific Research Publishing Inc. This work is licensed under the Creative Commons Attribution International License (CC BY 4.0).

http://creativecommons.org/licenses/by/4.0/

\begin{abstract}
Nutritional fads in the health and fitness world are constantly changing. Each new craze has its believers and critics. For the consumer, "what to believe" becomes a topic filled with uncertainty. This paper presents a systematic approach to understanding what consumers believe about the health messaging of "raw beverages". The paper presents both substantive results from US consumers, as well as demonstrates a general approach by which researchers can more deeply understand the consumer mind with respect to the specifics of health and wellness issues.
\end{abstract}

Keywords

Raw, Food, Beverages, Health, BiMiLeap

\section{Introduction-Why Today?}

An emerging fad in today's health and fitness world, quite simply, is the notion that "raw beverages" impart important health benefits. "Raw" as defined in this fad, means not only uncooked, but unfiltered, unpasteurized, and unsterilized. As consumers of Hollywood movies, we have all witnessed characters bending down and drinking straight from streams and lakes. However, what usually remains unseen, is the all-too-often outcome, illness, in the days and weeks which follow after drinking these untreated water sources.

The consumption of untreated water has recently increased due to a new fad of drinking "raw" water. The purveyors of raw water tout the added health bene- 
fits, such as it being the key to unlocking a perfect micro-biome balance. These consumers are currently paying up to $\$ 36.99$ for 2.5 gallons [1]. In addition to raw water, people are continuing to consume other raw products such as raw milk and raw juices.

\subsection{Pathogens}

The pathogens, or biological agents, responsible for morbidity and mortality from ingesting raw products include bacteria, viruses, and parasites. The most common pathogens are E. coli O157 and O111, Salmonella, Cryptosporidium and norovirus. Outbreaks have also been due to Vibrio cholerae, Clostridium botulinum, yeast and hepatitis A [2].

\subsection{Water Related Sickness}

Unfiltered, untreated water, even from the cleanest streams, can contain animal feces, spreading Giardia, which has symptoms such as vomiting and diarrhea and results in roughly 4600 hospitalizations a year. Hepatitis $A$, which resulted in 20 deaths in a California outbreak in 2017, can be spread through water if it isn't treated [3]. In addition, unfiltered drinking water may harbor many different, harmful microorganisms, for example viral (e.g.: Adenovirus, Norovirus, Hepatitis $A$ and E, etc.), bacterial (e.g.: E. coli, Salmonella enterica, Vibrio cholerae), protozoan (e.g.: Cryptosporidium hominis and parvum, Toxoplasma gondii), and fungal (e.g.: Aspergillus fumigatus, Candida albicans, Exophiala dermatitidis), respectively [4].

The CDC (Centers for Disease Control and Prevention) strongly recommends boiling, filtering or disinfecting water from streams and rivers before drinking it and calls modern water treatment techniques "One of the Ten Greatest Public Health Achievements of the $20^{\text {th }}$ Century" [2].

\subsection{Example of Raw Beverage Hazards-Raw Milk}

Raw milk is milk from any animal that has not been pasteurized to kill harmful bacteria. Also known as unpasteurized milk, raw milk can carry harmful bacteria, including Listeria monocytogenes, Brucella, Streptococcus, etc. and other organisms which are human pathogenic and in certain cases may cause death. Although it is possible to get foodborne illness from many kinds of foods, raw milk presents one of the highest risks [5]. It is important to note that even dairy products made from raw milk can cause severe health effects due to the unwanted microorganisms [6]. In addition to Listeria, a wide variety of other organisms occasionally found in raw milk make people sick. These germs include Brucella, Campylobacter, Cryptosporidium, E. coli, and Salmonella [5].

\subsection{Hazards of Raw Beverages-Raw Juices and Fruits}

During the past decade more than 1700 people in North America have been reported to fall ill after consuming juice and cider. Most of these outbreaks in- 
volved unpasteurized juices and ciders such as apple cider, orange juice, and lemonades. Other fresh fruit juice outbreaks included pineapple, carrot, coconut, cane sugar, banana, acai and mixed fruit juices. Despite the acidic environment of most fruit and vegetable juices, some pathogenic microorganisms are still able to contaminate and grow in the juice, and unprocessed juices are not processed to ensure that these microorganisms are killed or inactivated [5].

A recent study from Dar es Salaam city, Tanzania, identified the microbiological hazards of different raw fruit juices vended in different parts of the city. $E$. coli was found in $93 \%$ of the juices. Squeezing by a simple manual machine proved to be the most contaminating method of extraction. Furthermore, the type of juice influences the number of positive samples. For example, mixed fruit and mango juices were reported to be significantly more contaminated than passion and tamarind juices [7].

\section{Mind Genomics}

Despite the health risks, many celebrities and trend-setters have bought into these "pure" and "natural" raw products. Even though many scientists have evidence about the safety concerns of these beverages, there are still consumers who only focus on the advertised health benefits. This study focuses on the experimental analysis of messages about "raw", focusing on the responses of consumers to mixtures of messages which include aspects of "raw", and the quantification of the strength (and weakness) of the messages. The approach is known as Mind Genomics [8].

\subsection{Consumer Minds}

Efforts to uncover consumer minds have taken place since the first days of the consumerism. Rindell, Korkman, Gummerus, analyzed the role of brand images in consumer practices for uncovering brand strength. They identify and categorize consumer practice, proposing that consumers develop novel and personal practices related to brands [9].

Kniazeva investigated consumer perceptions of genetically modified food, and aims to explore what would make concerned consumers accept the idea. The focus was to uncover consumer mindsets through in-depth interviews [10].

Yarrow provides interesting insights about the new motivations and behaviors of shoppers, taking marketers where they need to be today. Yarrow focuses on what has come to be accepted as deeply psychological, often unconscious relationships, that people form with products, retailers, marketing communications, and brands, respectively [11].

\subsection{The Mind Genomics Approach}

In this paper we present a different approach to uncover the consumer's mind. Our approach is based on Mind Genomics, a new technology that tries to understand the minds of consumers by their reaction to messages [12]. Mind Genom- 
ics relies on the concepts of inter-market variability and horizontal segmentation, ideas pioneered by American market researcher, psychophysicist, and coauthor, Dr. Howard Moskowitz, who suggested that in any area where "opinion holds sway," one can discover different segments of consumer needs and predilections towards options that are provided. In other words, there are always different consumer mind-sets whenever there are topics calling for an opinion. We applied the analytical engine of Mind Genomics to explore the attitudes and mind-sets of consumers towards the notion of "raw", focusing our analysis on "raw beverages".

\section{Methods-A Systematic Approach}

\subsection{Background}

One of the benefits today, 2018, is the widespread computation power available in the form of applications (APPS), and executable on a smartphone. In keeping with the business objective of fast and simple, Moskowitz created an APP, BiMiLeap ${ }^{\oplus}$, which requires the researcher to ask four questions about a topic (e.g., raw beverages), provide four answers to each of the four questions, along with a rating scale. BiMiLeap presents respondents with unique sets of 24 test stimuli (vignettes), creates a model for each respondent relating the presence/absence of the elements to the rating, and then reveals the important versus irrelevant messages. The final analysis reveals different groups of respondents, so-called mind-set segments.

\subsection{Setup-Vignette}

The BiMiLeap program is set up with four questions and sixteen answers. Four answers link to each question. The respondent receives an invitation, either in an email or in social media. The respondent rates a unique set of 24 vignettes, each vignette dealing with raw food and raw beverages. Each vignette is constructed according to an underlying experimental design. Each respondent rates a unique set of 24 vignettes. An example of a vignette appears in Figure 1. The vignette

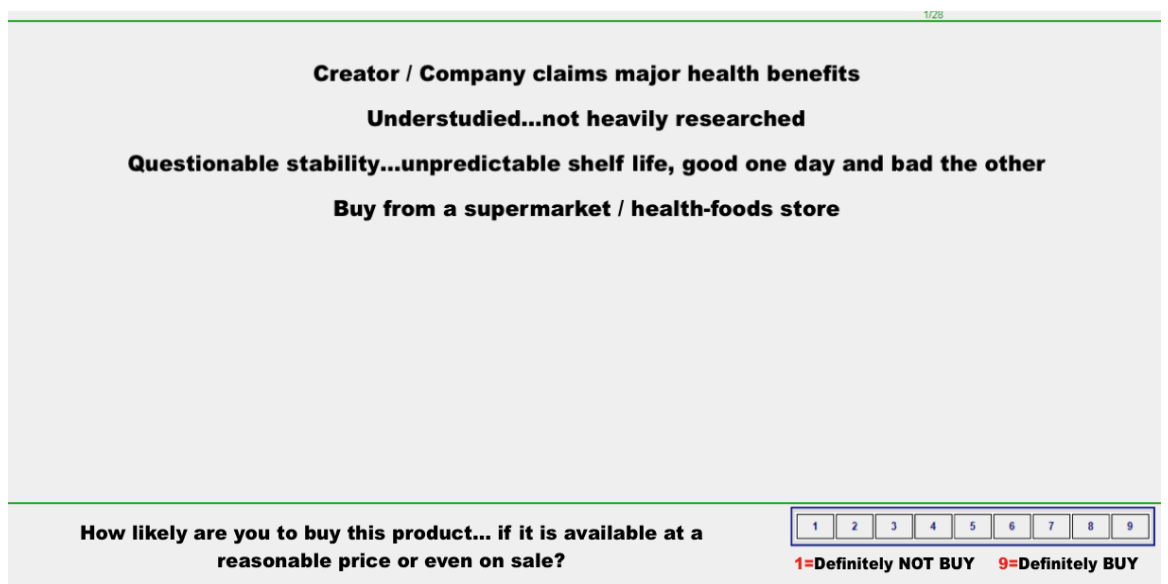

Figure 1. Example of vignette. 
comprises only answers, at most one answer from each question, but often a vignette may comprise 2 - 3 answers, not four. The respondent never sees the questions. The questions are only used to guide the creation of answers, the "messages" about the raw food and beverage.

\subsection{Converting from a Likert Scale to a Binary Scale}

The 9-point rating scale, a so-called Likert Scale, is difficult to understand. To make the results easier, we convert the ratings to a binary to a binary scale, with ratings of 1 - 6 converted to 0 , ratings 7 - 9 converted to 100. A small random number is added to each rating, to ensure that the ordinary least squares regression does not crash, when say the respondent assigned all 24 vignettes ratings of $1-6$, or all 24 vignette ratings of 7 - 9. This conversion makes the results easy to understand and interpret.

The binary rating from each respondent is then analyzed by OLS, ordinary least-squares regression, possible because the set of 24 vignettes had been created according to a main-effects experimental design. OLS relates the presence/absence of the 16 elements (coded as $0=$ absent, $1=$ present) to the binary ratings $(0=1$ $-6,100=7-9)$. The equation is expressed by the simple linear model, showing the contribution of each element to the binary rating:

$$
\text { Rating }=k_{0}+k_{1}(A 1)+k_{2}(A 2)+\cdots+k_{16}(D 4) \text {, }
$$

The elements are denoted as $A 1, A 2 \ldots D 4$, the additive constant is $\mathrm{k}_{0}$, and the 16 coefficients are denoted by $k_{n}$. These parameters of the regression equation (additive constant, 16 coefficients) all have meaning, as will be explained with the exposition of the results. The 16 coefficients are especially important because they reveal the contribution of the corresponding element to the rating [13].

\subsection{Model Analysis}

The results of the exercise comprise the above-mentioned 17 parameters for each regression model, one model estimated separately for each respondent. The analysis averages the corresponding coefficients for the relevant subgroup, whether total, gender, age groups and a third classification question, e.g., most important aspect of eating. The BiMiLeap APP then clusters the respondents into first two, and then three, four, five and then six clusters, based upon the pattern of the 16 coefficients. The additive constant is not considered in the clustering or segmentation.

Clustering algorithms are widely used in unsupervised pattern recognition to find groups and data points which show similar characteristics. The APP uses $k$-means clustering, a dynamic clustering algorithm, which uses the nearest mean approach to find the best possible cluster membership of each data point [14].

\section{Results}

\subsection{Clusters}

We now look at the results of clustering people, not into what raw beverages 
they like to drink, but rather what qualities of a raw beverage appeals to them when they consider messages about purchasing a product. These are the messages presented by the BiMiLeap APP, when the study was executed with consumer respondents. The clustering is based upon the pattern of the 16 coefficients. Clustering puts together respondents showing similar patterns of the 16 coefficients. The selection of the appropriate number of clusters or segments is subjective. Two good criteria are parsimony (fewer clusters are better than more clusters), and interpretability (the clusters should tell a reasonably simple, coherent "story").

Table 1 suggest three clusters, as named in the table, i.e., three mind-sets. The results show the desires of the different groups for the features of a raw beverage

Table 1. Table of results, showing what elements of a message about a raw beverage appeal to respondents. The group of 105 respondents divides into three unequally-sized segments, mind-sets, based upon the pattern of coefficients.

\begin{tabular}{|c|c|c|c|c|c|}
\hline & & & Risk-Averse & Germophobe & $\begin{array}{c}\text { Puddle } \\
\text { Drinkers }\end{array}$ \\
\hline & & Total Sample & Segment 1 of 3 & Segment 2 of 3 & Segment 3 of 3 \\
\hline & Base Size & 105 & 36 & 52 & 17 \\
\hline & Constant & 29 & 35 & 24 & 35 \\
\hline & Question: What are others' opinions? & & & & \\
\hline A1 & New fad ... public craze, mainstream media and celebrities buy in & -5 & 0 & -1 & -25 \\
\hline A2 & Creator/company claims major health benefits & -1 & -2 & 3 & -13 \\
\hline A3 & Some scientists call it dangerous & -11 & -4 & -13 & -19 \\
\hline \multirow[t]{2}{*}{ A4 } & Health and fitness community advocate the product & 1 & 4 & 5 & -18 \\
\hline & Question: What makes raw beverages different? & & & & \\
\hline B1 & Untreated ... pure, free of treatment chemicals & 2 & -8 & 6 & 10 \\
\hline B2 & Unfiltered ... still has original minerals and content & -1 & -14 & 5 & 11 \\
\hline B3 & Understudied ... not heavily researched & -4 & -16 & 2 & 2 \\
\hline \multirow[t]{2}{*}{ B4 } & Unregulated ... No U.S. Food and Drug Administration (FDA) approval & -2 & -17 & 8 & 0 \\
\hline & Question: What risks and benefits are involved? & & & & \\
\hline $\mathrm{C} 1$ & Risk of harmful bacteria, viruses and parasites & -9 & -6 & -18 & 10 \\
\hline $\mathrm{C} 2$ & Good for you, good for the environment ... green, healthy & 9 & 7 & 9 & 16 \\
\hline $\mathrm{C} 3$ & Part of an all-natural diet ... no artificial ingredients & 3 & -6 & 5 & 18 \\
\hline \multirow[t]{2}{*}{$\mathrm{C} 4$} & $\begin{array}{l}\text { Questionable stability ... unpredictable shelf-life, good one day and bad } \\
\text { the other }\end{array}$ & -7 & -2 & -16 & 11 \\
\hline & Question: Where can you obtain the beverage? & & & & \\
\hline D1 & Source it yourself or from your friends ... local & 1 & 1 & 1 & 1 \\
\hline D2 & Buy from a supermarket/health-foods store & -1 & 1 & -3 & 0 \\
\hline D3 & Purchase from a farmer's market & 5 & 6 & 6 & 1 \\
\hline D4 & Buy it online & 2 & 3 & 4 & -7 \\
\hline
\end{tabular}


that one would buy (Table 1). The additive constant corresponds to the conditional probability of saying that one would buy the raw product in the absence of elements. The additive constant is clearly an estimated parameter, serving as a computed baseline. Each element generates a coefficient, which is the additive additional interest in buying the raw product. The contribution can be positive (increases the interest when inserted into the vignette), or the contribution can be negative (decreases the interest when inserted into the vignette). The elements are combined with the additive constant, with the sum showing the total probability of buying interest.

The vignette should include at least one answer, and at most four answers, from the different questions. A good rule of thumb from the Mind Genomics studies are that coefficients exceeding \pm 7.51 correspond to elements that co-vary with external behaviors. This value of \pm 7.51 applies to the total panel, to subgroups identified either from the classification, or to groups or clusters emerging from mind-set segmentation.

\subsection{Analyzing the Results}

As shown in Table 1, three segments were created from this analysis. Segment 1 consists of $34 \%$ of the sample respondents. This group has a risk-aversion mindset, wanting treated, filtered, studied, and regulated products. They are cautious about what they put into their body, avoiding harmful substances whenever possible. Segment 2 are the germophobes and consist of roughly $50 \%$ of the sample. This group cares about their environmental impact while also steering clear of sickness causing factors such as bacteria growth and unstable shelf life. Segment 3 comprises individuals who don't care about other people's opinions, the so-called puddle drinkers. Segment 3 comprises $16 \%$ of this sample. Segment 3 will eat or drink anything if they can be convinced that the product has "natural," which they associate with health benefits. Segment 3 , the most extreme, are those drawn to all natural, homegrown raw foods, which they believe will lower the environmental impact produced by today's agriculture.

\section{Discussion}

\subsection{Applying the Results}

Now we venture back to those celebrities and trend-setters who have bought into the craze of raw products. This group appears to be members of the three mind-sets, "The Puddle Drinkers." They understand the risks of untreated and unfiltered products. On the other hand, they do not appear to care about other opinions, seemingly focused only on the benefits.

\subsection{Key Messages-Marketing Opportunity, Social Responsibility}

Mind Genomics is the actionable, scalable, and empirical means for discovering what to say, how to say it, and to whom. It is truly powerful because it grants the ability to approach a consumer with the exact message that brings them closer to 
"yes". To appeal to consumers, one needs to include the proper words in one's message. If one is to incorporate good health practices with today's runaway success of "raw", it might be a good idea to incorporate the proper messages from Mind Genomics into the messages which talk about better health practice. For example, a message directed to the "Puddle Drinkers" should focus on "low risk, high reward, all natural, live symbiotically with the earth." A message directed to the "Germophobes" should focus on "absence of harmful bacteria, good for you and the environment." By directly targeting each segment with the proper message, the company or the health agency might well capture the attention and interest of the consumer, and increase their chances of compliance.

\section{Conclusion}

This study has demonstrated the use of Mind Genomics, experimental analysis of ideas, executed inexpensively and quickly with a smartphone APP, BiMiLeap. Three mind-types emerged through this systematic approach: Risk-averse, Germophobes, and Puddle drinker, respectively. These three groups differ: Those who respond to messages about low risk of harmful content, those who respond with fear to messages about food related sickness, and those who say that will drink or eat anything given that there are benefits. These results suggest a need to understand AND communicate proper information about the microbiological hazards of raw products. Consumer education needs to take into account both the message to be sent, and the nature of the person to whom the message is intended to influence. The right message may work. The wrong message may be technically correct, but may end up being ignored, or even actively rejected.

\section{Acknowledgements}

We sincerely thank the BiMiLeap staff for allowing the use of their app and their devotion to creating learning opportunities for all.

\section{References}

[1] Nellie, B. (2017) Unfiltered Fervor: The Rush to Get off the Water Grid. The New York Times. www.nytimes.com/2017/12/29/dining/raw-water-unfiltered.html

[2] Centers for Disease Control and Prevention (2017) Food Safety. www.cdc.gov/foodsafety/rawmilk/raw-milk-questions-and-answers.html

[3] (2018) San Diego Hepatitis A Outbreak. Hepatitis A, Health \& Human Services Agency. www.sandiegocounty.gov/content/sdc/hhsa/programs/phs/community_epidemiolo gy/dc/Hepatitis_A.html

[4] Ashbolt, N.J. (2015) Microbial Contamination of Drinking Water and Human Health from Community Water Systems. Current Environmental Health Reports, 2, 95-106. https://doi.org/10.1007/s40572-014-0037-5

[5] Center for Food Safety and Applied Nutrition (2017) Juice-Guidance for Industry: Juice HACCP Hazards and Controls Guidance First Edition. US Food and Drug Administration Home Page, Center for Food Safety and Applied Nutrition. 
https://www.fda.gov/Food/GuidanceRegulation/GuidanceDocumentsRegulatoryInf ormation/Juice/ucm072557.htm

[6] Verraes, C., Vlaemynck, G., Van Weyenberg, S., De Zutter, L., Daube, G., Sindic, M., Uyttendaele, M. and Herman, L. (2015) A Review of the Microbiological Hazards of Dairy Products Made from Raw Milk. International Dairy Journal, 50, 32-44. https://doi.org/10.1016/j.idairyj.2015.05.011

[7] Simforian, E., Nonga, H.E. and Ndabikunze, B.K. (2015) Assessment of Microbiological Quality of Raw Fruit Juice Vended in Dar es Salaam City, Tanzania. Food Control, 57, 302-307. https://doi.org/10.1016/j.foodcont.2015.04.033

[8] Veljko, M. and Salom, J. (2016) Mind Genomics A Guide to Data-Driven Marketing Strategy. Springer, Berlin, 2191-5482.

[9] Rindell, A., Korkman, O. and Gummerus, J. (2011) The Role of Brand Images in Consumer Practices: Uncovering Embedded Brand Strength. Journal of Product \& Brand Management, 20, 440-446. https://doi.org/10.1108/10610421111166586

[10] Kniazeva, M. (2008) Marketing "Frankenfood": Appealing to Hearts or Minds? Journal of Food Products Marketing, 11, 21-39. https://doi.org/10.1300/J038v11n04_03

[11] Yarrow, K. (2014) Decoding the New Consumer Mind: How and Why We Shop and Buy. John Wiley, ISBN: 978-1-118-64768-4.

[12] Gofman, A. (2012) Putting RDE on the R\&D Map: A Survey of Approaches to Consumer-Driven New Product Development. In: Gofman, A. and Moskowitz, H.R., Eds., Rule Developing Experimentation: A Systematic Approach to Understand \& Engineer the Consumer Mind, Bentham Books, Sharjah, 72-89.

[13] Hastie, T., Tibshirani, R. and Friedman, J. (2011) The Elements of Statistical Learning. 2nd Edition, Springer, New York.

[14] Hong, X., Wang, J. and Qi, G. (2014) Comparison of Spectral Clustering, K-Clustering and Hierarchical Clustering on e-Nose Datasets: Application to the Recognition of Material Freshness, Adulteration Levels and Pretreatment Approaches for Tomato Juices. Chemometrics and Intelligent Laboratory Systems, 133, 17-24. https://doi.org/10.1016/j.chemolab.2014.01.017 